\title{
Which factors affect the success of pediatric PCNL? Single center experience over 20 years
}

\author{
Volkan Izol ${ }^{1}$, Nihat Satar ${ }^{1}$, Yildirim Bayazit ${ }^{1}$, Fatih Gokalp ${ }^{2}$, Nebil Akdogan ${ }^{1}$, \\ Ibrahim Atilla Aridogan ${ }^{1}$ \\ ${ }^{1}$ Department of Urology, Faculty of Medicine, University of Çukurova, Adana, Turkey; \\ ${ }^{2}$ Clinic of Urology, Osmaniye Government Hospital, Osmaniye, Turkey.
}

\begin{abstract}
Summary Objective: We aimed to investigate the impact of surgeons' experience on pediatric percutaneous nephrolithotomy (PCNL) outcomes.

Materials and methods: Between June 1997 and June 2018, 573 pediatric patients with 654 renal units underwent PCNL for renal stone disease by senior surgeons. Data were divided into two groups, group-1 $(n=267)$, first ten years period, group-2 $(n=387)$; second ten years period.

Results: Mean \pm SD age of patients was $7.6 \pm 4.9$ (1-17) years. The stone-free rates (SFR) assessed after 4 weeks were $74.9 \%$ vs. $83.4 \%$ in group-1 vs. group-2, respectively $(p=0.03)$. The mean operation time, fluoroscopy time, and the number of patients requiring blood transfusion significantly decreased in group $2(100.4 \pm 57.5$ vs. $63.63 \pm 36.3,12.1 \pm 8.3$ vs. $8.3 \pm$ 5.4 , and $24.3 \%$ vs. $2.9 \% ; p<0.001, p<0.001$, and $p=0.002$ in group-1 versus group-2, respectively). On multivariate analysis, increasing stone size increased operation time ( $p<0.001)$, fluoroscopy time $(p<0.001)$, intraoperative and postoperative blood transfusion rates ( $p=0.006$ and $p=$ 0.018 , respectively), and hospital stay $(p=0.002)$ but was not associated with change of glomerular filtration rate (GFR) $(p=0.71)$. Sheath size also correlated with increased fluoroscopy time $(p<0.001)$, operation time $(p<0.001)$, intraoperative blood transfusion $(p<0.001)$ and hospital stay, but sheath size did not affect postoperative blood transfusion ( $p=0.614)$ or GFR change $(p=0.994)$.

Conclusions: The percutaneous nephrolithotomy (PCNL) is a minimally invasive procedure and is well accepted because of its lower complication rate and high efficiency for pediatric patients. Stone and sheath size are predictive factors for blood loss and hospital stay. During 20 years, our fluoroscopy time, operation time, blood loss, and complication rates decreased, and stone-free rate increased.
\end{abstract}

KEY WORDS: Pediatric; Percutaneous; Urinary calculi; Endourology.

Submitted 24 May 2020; Accepted 30 July 2020

\section{INTRODUCTION}

Pediatric stone-disease is widespread in developing countries and Turkey (1). The surgical management of stone disease has changed because of technological advances in recent years. For renal stones smaller than 2 $\mathrm{cm}$, extracorporeal shock wave lithotripsy (SWL) and ret- rograde intrarenal surgery (RIRS) are the treatments of choice in children (2). Additionally, over the last two decades, percutaneous nephrolitotomy (PCNL), with low complications and high success rates, has become the standard treatment of choice for kidney stones $>2 \mathrm{~cm}$ and is an alternative procedure for stone size between 1 $2 \mathrm{~cm}$ at lower pole $(2,3)$.

PCNL is an effective and safe procedure in pediatric patients (4-7). Nevertheless, serious complications such as bleeding requiring transfusion, organ injuries, pneumothorax, infection, and sepsis, still have been reported for this procedure (7). There are a few factors identified as affecting complications, including stone size, sheath size, number of punctures, presence of hydronephrosis, and prolonged operation time $(8,9)$. Recent studies of stone disease treatment showed increased success rates, and also decreased complication rates in association with increase of expertise in high volume centers $(7,8)$. In this retrospective study, we aimed to evaluate the impact of surgeons' experience on complication rates, success rates, and the management of complications along a period of more then 20 years.

\section{Materials AND METHOdS}

Between June 1997 and June 2018, 573 pediatric patients with a total of 654 renal units underwent PCNL for renal stone disease. The patients with bilateral kidney stones were treated with staged procedures. All patients were assessed preoperatively with excretory urography, renal ultrasound, and/or non-enhanced spiral computerized tomography, and urine was collected for culture analysis before surgery. Written informed consent was achieved for all participants. After approvel by Cukurova University Ethics Committee, June 2018/78, preoperative data were obtained, including gender, age, operation time, sheath size, laterality, stone burden, hematocrit, and serum creatinine. The stone burden was calculated by the stone surface area formula.

Intraoperative and postoperative data were obtained including pre/postoperative variation of glomerular filtration rate (GFR) (calculated with Cockcroft Gault equations), drop of hemoglobin levels, transfusion rate, complications according to the Clavien classification, operative time, length of hospital stay and stone-free rate (SFR) $(10,11)$.

\footnotetext{
No conflict of interest declared.
} 
Additionally, we divided the data into two groups. In group-1, PCNL was performed in the first ten years period (1997-2007); in group-2, PCNL was performed in the second ten years period from 2008 to the present.

\section{Surgical technique}

All procedures were performed by four experienced surgeons who had been working for at least 20 years in our clinic and staff surgeons under supervision of mentors. All patients received prophylactic antibiotics preoperatively during anesthesia induction. After the placement of a 5 Fr ureteral open-end catheter, patients were positioned in a prone position with proper constructional support by silicone rolls. The pelvicalyceal system was visualized by injecting the radiographic contrast dye through the ureteric catheter. The collecting system was punctured with a needle under fluoroscopy, a guidewire was inserted, and then the urinary tract was dilated with metal or Amplatz dilatators. After placement of an 18-30 Fr sheath, a 15Fr-19Fr rigid nephroscope or $9.5 \mathrm{Fr}$ rigid ureteroscope was inserted into the collecting system. Stones were fragmented with a pneumatic lithotripter or laser and retrieved with rigid or flexible forceps or graspers. A 10 Fr nephrostomy tube was left in place if needed. We preferred the tubeless technique for selected cases such as those with short operation time, a single puncture for a tract, undamaged pelvicalyceal system, no major bleeding or residual stones at the end of the procedure.

On the second postoperative day, the patient underwent antegrade pyelography, or the nephrostomy tube was clamped if there was no residual fragment or extravasation detected by imaging. Then, the nephrostomy tube was extracted. Patients were discharged after drainage from the nephrostomy tract was stopped. If the drainage from the nephrostomy tract continued longer than seven days, it was defined as prolonged drainage, and we inserted a double J stent. When drainage was stopped for several days, patients were discharged.

\section{Follow up}

The first visit for follow up was done at 4-6 weeks after discharge, including urinalysis, metabolic examination of 24-hour urine specimens, and urinary ultrasonography. Stone free rates (SFR) were evaluated at 4 week follow. We reported the result as a failure in presence of any asymptomatic residual stone fragment $>4 \mathrm{~mm}$ at 4 week follow up. We confirmed the result by using intravenous urography in the first-year period and nonenhanced computerized tomography in the second ten years period.

\section{Statistical analysis}

SPSS, version 20.0, was used to perform statistical analysis. The Kolmogorov Smirnov test was used in the numerical computations provided the assumption of a normal distribution. For comparing the categorical measurements between the groups, the chi-square test was used. Mann-Whitney U, chi-square, ANOVA, and logistic regression were used for multivariate analysis. Statistical significance was defined as a p-value of less than 0.05 .

\section{RESULTS}

\section{Demographic data}

Five hundred seventy-three pediatric patients with 654 renal units were evaluated in the study. The mean \pm SD age of patients was $7.6 \pm 4.9$ years. The mean stone burden was $371.8 \pm 459.4$ (95\% CI: $343.0-415.5) \mathrm{mm}^{3}$. There were $343(52.5 \%)$ renal units with a single calyceal stone and $311(47.5 \%)$ patients with multiple calyceal or staghorn stones. SFR was found $81.4 \%$ $(n=533)$. The mean hospital stay was $4.6 \pm 4.2$ days. In our practice, the usual hospital course for PCNL is 3-4 days in pediatric patients, but $173(26.4 \%)$ patients stayed longer ( 7 to 27 days) due to leakage drainage, bleeding, infections, or other complications (Table 1).

\section{Operative outcomes}

On multivariate analysis, the perioperative parameters such as fluoroscopy time, bleeding, and operation time were associated with stone size and sheath size.

Increasing stone size increased operation time $(p<0.001)$, fluoroscopy time $(p<0.001)$, intraoperative and postoperative blood transfusion rate $(\mathrm{p}=0.006$ and $\mathrm{p}=0.018$, respectively), and hospital stay $(\mathrm{p}=0.002)$ but was not associated with GFR change $(\mathrm{p}=0.71)$.

Sheath size also correlated with th parameters and increased fluoroscopy time $(p<0.001)$, operation time $(p<0.001)$, intraoperative blood transfusion $(p=0.002)$ and hospital stay, but sheath size did not affect postop-

Table 1.

Demographic data of patients.

\begin{tabular}{|c|c|}
\hline & Value \\
\hline No. of patients & 654 \\
\hline$\overline{\text { Age (years) }}{ }^{\mathrm{a}}$ & $7.6 \pm 4.9$ \\
\hline \multicolumn{2}{|l|}{ Gender $^{b}$} \\
\hline M & $167(62.5 \%)$ \\
\hline $\mathrm{F}$ & $100(37.5 \%)$ \\
\hline \multicolumn{2}{|l|}{ Laterality $^{b}$} \\
\hline Left & $326(49.8 \%)$ \\
\hline Right & $328(50.2 \%)$ \\
\hline \multicolumn{2}{|l|}{ Site of stone $e^{b}$} \\
\hline Single calyx & $343(52.5 \%)$ \\
\hline Multiple calyces & $311(47.5 \%)$ \\
\hline Operation time ${ }^{\mathrm{a}}$ & $78.3 \pm 49.0$ \\
\hline Stone free rate ${ }^{b}$ & $533(81.4 \%)$ \\
\hline
\end{tabular}

Table 2.

Complications causing prolonged hospital stay.

\begin{tabular}{|lccc|}
\hline & $\begin{array}{c}\text { Group 1 } \\
(\mathbf{1 9 9 7 - 2 0 0 7 )}\end{array}$ & $\begin{array}{c}\text { Group 2 } \\
\mathbf{( 2 0 0 8 - 2 0 1 8 )}\end{array}$ & p value \\
\hline Infection and fever & $26(9.7 \%)$ & $15(3.8 \%)$ & 0.02 \\
\hline Leakage drainage & $10(3.7 \%)$ & $11(2.8 \%)$ & 0.52 \\
\hline Requiring stenting & $6(2.2 \%)$ & $7(1.8 \%)$ & 0.69 \\
\hline Colon perforation & $2(0.7 \%)$ & $1(0.2 \%)$ & 0.79 \\
\hline Collecting system perforation & $1(0.3 \%)$ & $4(1.0 \%)$ & 0.34 \\
\hline Bleeding & $78(29.2 \%)$ & $22(5.6 \%)$ & $<0.001$ \\
\hline Requiring blood transfusion & $65(24.3 \%)$ & $11(2.8 \%)$ & $<0.001$ \\
\hline Transcathateter angiography & $2(0.7 \%)$ & $1(0.2 \%)$ & 0.36 \\
\hline All data was presented as n (\%). & & & \\
\hline
\end{tabular}


erative blood transfusion $(p=0.614)$ or GFR change $(\mathrm{p}=0.994)$. Furthermore, prolonged operation time increases fluoroscopy time $(\mathrm{p}<0.001)$, intraoperative and postoperative blood transfusion $(\mathrm{p}<0.001$ and $p=0.008$, respectively), and hospital stay $(p<0.001)$, but not associated with GFR change $(\mathrm{p}=0.55)$.

Stone size and sheath size were not associated with postoperative changes of GFR. SFR was correlated with operation time (B: $-0.013, \mathrm{p}=0.02)$ (Table 3 ). Table 4 shows the comparison of the outcomes of two subgroups of patients treated in two different period of time using different-sized instruments. Mean operation time, fluoroscopy time, and blood transfusion rate were signifi-

\section{Table 3.}

Multivariate analysis of SFR compared to demographic and perioperative parameters.

\begin{tabular}{|lccccc|}
\hline & \multicolumn{2}{c}{ Unstandardized coefficients } & \multicolumn{2}{c|}{ 95\% Confidence interval } \\
& $\mathbf{B}$ & Std. error & $\mathbf{p}$ & Lower & Upper \\
\hline Age & .047 & .056 & .399 & .939 & 1.170 \\
\hline Gender &. .024 & .300 & .936 & .543 & 1.756 \\
\hline Laterality &. .264 & .301 & .380 & .426 & 1.385 \\
\hline Weight &. .017 & .014 & .226 & .956 & 1.011 \\
\hline Stone size &. .001 & .001 & .108 & .998 & 1.000 \\
\hline Operation time & -.013 & .006 & .021 & .976 & .998 \\
\hline Fluoroscopy time & .000 & .000 & .781 & .999 & 1.001 \\
\hline Sheath size & .079 & .070 & .259 & .944 & 1.241 \\
\hline Constant & 1.367 & 1.614 & .397 & & \\
\hline *Depended variable was SFR. & & & & & \\
\hline
\end{tabular}

Table 4.

Difference characteristics of two chronological groups.

\begin{tabular}{|c|c|c|c|}
\hline & $\begin{array}{c}1997-2007 \\
(n=267)\end{array}$ & $\begin{array}{r}2008-2018 \\
(n=387)\end{array}$ & $p$ value \\
\hline Age (years) $)^{2}$ & $8.5 \pm 4.9$ & $6.9 \pm 4.7$ & \\
\hline \multicolumn{4}{|l|}{ Gender $^{b}$} \\
\hline M & $167(62.5 \%)$ & $210(54.2 \%)$ & \\
\hline $\mathrm{F}$ & $100(37.5 \%)$ & 177 (45.8\%) & \\
\hline \multicolumn{4}{|l|}{ Sideb } \\
\hline Left & $128(47.9 \%)$ & $198(51.1 \%)$ & \\
\hline Right & $139(52.1 \%)$ & $189(48.9 \%)$ & \\
\hline Stone sizea $\left(\mathrm{mm}^{2}\right)$ : & $480.8 \pm 380.0$ & $295.8 \pm 196.5$ & $p<0.001$ \\
\hline Sheath sizea: & $27.87 \pm 2.8$ & $24.41 \pm 2.6$ & $p<0.001$ \\
\hline GFR change (mg/d)): & $2.6 \pm 0.2$ & $3.8 \pm 0.3$ & $p=0.425$ \\
\hline Serum creatinine changea $(\mathrm{mg} / \mathrm{dll})$ : & $0.013 \pm 0.0$ & $0.001 \pm 0.0$ & $p=0.717$ \\
\hline Operation timea (min): & $100.4 \pm 57.5$ & $63.63 \pm 36.3$ & $p<0.001$ \\
\hline Fluoroscopy timea (min): & $12.19 \pm 8.3$ & $8.31 \pm 5.4$ & $p<0.001$ \\
\hline Hemorrhage requiring transfusionb: & $65(24.3 \%)$ & $11(2.9 \%)$ & $p=0.002$ \\
\hline \multicolumn{4}{|l|}{ Stone free rates ${ }^{\mathrm{b}}$} \\
\hline SF & $200(74.9 \%)$ & $323(83.4 \%)$ & $p=0.03$ \\
\hline Failure & $64(24.2 \%)$ & 56 (14.8\%) & \\
\hline Nephrostomy removal time (day): & 2.93 & 2.57 & $p=0.133$ \\
\hline \multicolumn{4}{|l|}{ Postoperative Complications: } \\
\hline Clavien Dindo ${ }^{b}$ & & & $p=0.842$ \\
\hline 1 & $39(14.6 \%)$ & $26(6.7 \%)$ & $p=0.001$ \\
\hline 2 & $69(25.8 \%)$ & $15(3.8 \%)$ & $p<0.001$ \\
\hline $3^{\mathrm{a}}$ & $8(2.9 \%)$ & $8(2.0 \%)$ & $p=0.45$ \\
\hline $3^{b}$ & $1(0.3 \%)$ & $4(1.0 \%)$ & $p=0.34$ \\
\hline $4^{a}$ & $2(0.7 \%)$ & $1(0.2 \%)$ & $p=0.36$ \\
\hline 5 & 0 & 0 & \\
\hline
\end{tabular}

cantly lower in group-2 ( $p<0.001, \mathrm{p}<0.001$, and $\mathrm{p}=0.002$, respectively). However, stone volume and sheath size also significantly decreased in group-2 $(\mathrm{p}<0.001)$. SFR were $74.9 \%$ and $86.4 \%$ in group- 1 and group-2, respectively $(\mathrm{p}=0.03)$.

Reoperation rates were 18 (6.7\%) versus $11(2.9 \%)$ in group-1 vs. group- 2 , respectively $(\mathrm{p}=0.01)$. There was no significant change between preoperative and postoperative mean GFR in both groups $(2.51 \pm 0.2 \mathrm{~mL} / \mathrm{min}$ and $3.83 \pm 0.3 \mathrm{~mL} / \mathrm{min} ; \mathrm{p}=0.584, \mathrm{p}=0.536$ in group1 and group- 2 , respectively).

The most common complications were low-grade complications, grade I (21.3\%), and grade II (29.6\%). Grade I and II complications were significantly higher group-1 than group-2 ( $p=0.001$ and $p<0.001)$. Grade III-a and III-b complications were seen in $16(2.4 \%)$ and $5(0.7 \%)$ patients, respectively, and there was no significant difference between groups $(p=0.45, p=0.34)$.

\section{Complications and management}

The most common complication was bleeding in both groups $(29.2 \%$ and $5.6 \%$ in group-1 and group-2, respectively). Rates of hemorrhages requiring transfusions were significantly different between the two groups (24.3\% and $2.9 \%$ in group-1 and group-2, respectively, $p=0.02$ ). Three patients underwent angiography for severe bleeding after the procedure, and a ten-year-old child underwent embolization for a pseudoaneurysm of the kidney. Twenty-six (9.7\%) children in group-1 and fifteen (3.8\%) in group-2 had a fever after PCNL $(p=0.07)$. Patients took different antibiotic regimens after a positive urine culture.

Twenty-one patients were followed up for prolonged drainage after removal of the nephrostomy tube. Thirteen patients, six (2.2\%) in group-1 and seven $(1.8 \%)$ in group-2, required double-J stenting. One patient required stenting for perirenal urinoma that resolved spontaneously. Collecting system perforations were seen in five patients, one $(0.3 \%)$ in group-1 and four $(1.0 \%)$ in group- 2 . Three patients had a long-staying nephrostomy tube from four to seven days, which was extracted after no extravasation was demonstrated at anterograde pyelography. Two cases required open surgery, one underwent pyeloplasty for damaged ureter-pelviv junction (UPJ) and the other underwent a primary repair of the renal pelvis.

Two $(0.7 \%)$ patients in group- 1 and one $(0.2 \%)$ in group2 had a colon perforation. One of them was treated with open surgery and a colostomy by the pediatric surgeon. The colostomy was closed after three months when anastomosis was done successfully. Two patients were treated conservatively, by withdrawal of the nephrostomy tube outside the kidney into the colon as a percutaneous colostomy tube and by insertion of a double-J ureteral stent for separating nephron-colic communication. Patients took intravenous broad-spectrum antibiotics and total parenteral nutrition. After 7-10 days, the patients started receiving oral feeding. The tube was removed after complete healing of the colon. One month after, the $\mathrm{J}$ stent was extracted under control of retrograde pyelography. One child underwent nephrectomy for a nonfunctioning kidney that was not producing urine in the 
postoperative days. Histopathological evaluation revealed xanthogranulomatous pyelonephritis.

\section{Discussion}

Pediatric stone disease is an important issue, and the critical point for preventing recurrence and relative complications after surgery is stone clearance. There still is not a consensus on describing stone-free rates. SFR varied in the studies due to the differences between pediatric and adult kidney anatomy, variable stone size, use of different-sized instruments, and inclusion or exclusion of clinically insignificant residual fragment (CIRF) cases. A recent study by Çıtamak et al. presented their results at four years intervals over 17 years, and stone-free rates were $73.5 \%, 68.1 \%, 75.5 \%$, and $74.0 \%$, with no significant difference among the groups $(\mathrm{p}=0.65)(12)$. Our study showed that our increasing clinical experience and use of small instruments incresed SFR after pediatric PCNL.

Our SFR was significantly higher in group-2 than group-1 and reoperation rates were similar into groups. Similar to our study, Yadav et al. study reported that stone-free rates were increased over 15 years from $84.6 \%$ to $89.9 \%$ (13). The early pediatric PCNL series were performed using adult-size instruments. The improvements in the devices and techniques of PCNL facilitated urologists to perform this procedure $(14,15)$. In our study, the mean sheath size decreased from $28 \mathrm{Fr}$ to $24 \mathrm{Fr}$ over the years. Bilen et al. compared three different sized nephroscope, and stone-free rates were $69.5 \%, 80 \%$, and $90 \%$ in the $26 \mathrm{Fr}$, $20 \mathrm{Fr}$, and miniperc groups, respectively $(\mathrm{p}<0.005)$.

The authors emphasized that the 26Fr and 20Fr groups include more patients with semi-staghorn and staghorn calculi (16). A novel systematic review showed that the minimal invasive PCNL (micro-ultra mini) success rate ranged between $85-100 \%$ (17)

In our study, the total complication rate (Clavien I-IV) was $28.4 \%$, and there was no significant difference between the two groups. Similar results were found in the CROES study which showed that the complication rate was $23.3 \%$ (7). Novel research showed the complications were decreased over the years $(33.8 \%, 23.6 \%$, $19.6 \%$, and $11.5 \%$, respectively, for every four years, $\mathrm{p}<0.001)(12)$. In the literature, the studies compared PCNL complications in children using different instruments, and the complications were graded according to the modified Clavien system, and similar to our research, most of the complications were grade I and II $(5,18,19)$. Ozden et al., in a study of 100 patients using pediatric instruments, reported an overall complication rate of $25 \%$ (18). Another study by Guven et al. reported that the total complication rate was $29.1 \%(\mathrm{n}=140)$, and there was no significant difference between pediatric and adult instruments $(p=0.52)(5)$. Additionally, Bilen et al. also showed that complication rates were not significantly different in their study comparing three different sized instruments (16). Mishra et al. examined the outcomes of miniperc (MPCNL) versus standard conventional PCNL and concluded that the MPCNL is significantly superior to PCNL in bleeding and hospital stay time (19). Similar to our study, novel papers showed that recently presented small size percutaneous accesses, such as MPCNL and micro percutaneous method (Micro-PCNL), are safer than classic PCNL in children $(2,20)$. Our study demonstrated that there had been no significant change in the treatment of complications until today, but we became less invasive due to our increased knowledge of complications.

The most common complications were bleeding and fever. Types and frequencies of complications were similar to those in adults $(8,17,21)$. Zeren et al. study showed correlation of intraoperative bleeding with operation time, sheath size, and stone size (8). Our research demonstrated that stone size and operation time correlated with intraoperative and postoperative blood transfusion. We also found that a larger sheath size is related to higher intraoperative blood transfusion rates and longer hospital stay. Similar to our study, Altintaş et al. study compared three different sheath sizes (17 Fr, 24 $\mathrm{Fr}$, and $26 \mathrm{Fr}$ ) showing that sheath size was related to increased intraoperative bleeding although there was no significant difference of preoperative and postoperative creatinine levels $(p=0.873)(22)$. However, controversial to our study, the literature also reported that sheath size did not affect transfusion rates $(12,16,23)$.

Fever was the second common complication in our study. Previous studies reported that the postoperative fever rate was approximately present in 29\% $(18,21)$. Bayrak et al. reported that postoperative fever rates in their study, comparing children to adults, were 5.4\% and $5.6 \%$, respectively (24). Celik et al. showed that postoperative fever rates were similar and reported rates of $5.9 \%$ and $6.8 \%$ using pediatric (18Fr) and adult-sized (24Fr) nephroscope, respectively (25).

In our study, postoperative fever rates were not significantly different in the two groups and similar to published literature.

Herein, we compared different-sized instruments and experience time and found that PCNL is an operatordependent procedure, which improves its results, presumably due to increased operator experience and the involvement of a team with substantial prior knowledge. Furthermore, our results depend on advances to technology that shifted the management of stone disease to minimal invasive modalities.

We realized that we treated smaller stones in the second ten years period.

The main reasons for this finding are:

- Increasing of diagnosis of patients with small stone size due to the novel radiological tools and easier access to health care services.

- In our clinic, use of a ESWL machine that uses fluoroscopy for stone localization (with no ultrasoundguided ESWL machine around the region)

- Technological advancement with development of small instruments for urologic endoscopic procedures.

Limitations of the study included its retrospective nature and the absence of a metabolic evaluation and chemical analysis of the composition of the stone. The second significant limitation was insufficient data of follow up, especially on group-1 and no standardization of timing or use of imaging tools at follow up. Another limitation of the study was the performance of percutaneous pro- 
cedures by more surgeons using different instruments at different periods being the experience of each surgeon a possible source of bias. However, a strenght of the study is that the data and results were obtained from a single large volume center.

\section{Conclusions}

Our study showed that the patient cohort became younger with smaller stone sizes over time.

Concordantly, the fluoroscopy time, operation time, blood loss, and complications rates were decreased, and stone free-rates were increased with use of smaller instruments. The stone and sheath size are major factors to predict blood loss and hospital stay.

\section{REFEREnCES}

1. Bartosh SM. Medical management of paediatric stone disease. Urol Clin North Am. 2004; 31:575-87.

2. Türk C, Neisius A, Petřik A, et al. EAU Guidelines on interventional treatment for urolithiasis. EAU Guidelines. Edn. presented at the EAU Annual Congress London 2018. ISBN 978-94-92671-01-1.

3. Matlaga BR, Kim SC, Lingeman JE. Improving outcomes of percutaneous nephrolithotomy: access. Eur Urol. EAU Update Ser. 2005; 3:37-43

4. Woodside JR, Stevens GF, Stark GL, et al. Percutaneous stone removal in children. J Urol. 1985; 134:1166-7.

5. Guven S, Istanbulluoglu O, Gul U, et al. Successful percutaneous nephrolithotomy in children: multicentre study on current status of its use, efficacy and complications using Clavien classification. J Urol. 2011; 185:1419-24.

6. Unsal A, Resorlu B, Kara C, et al. Safety and efficacy of percutaneous nephrolithotomy in infants, preschool age, and older children with different sizes of instruments. Urology. 2010; 76:247-52.

7. Guven S, Farttini A, Onal B, et al. Behalf of the CROES PCNL Study Group. Percutaneous nephrolithotomy in children in different age groups: data from the Clinical Research Office of the Endourological Society (CROES) Percutaneous Nephrolithotomy Global Study. BJU International. 2012; 111:148-56.

8. Zeren S, Satar N, Bayazit Y, et al. Percutaneous nephrolithotomy in the management of pediatric renal calculi. J Endourol. 2002; 16:75-8.

9. Desai M. Endoscopic management of stones in children. Curr Opin Urol. 2005; 15:107-12.

10. Cockcroft DW, Gault MH. Prediction of creatinine clearance from serum creatinine. Nephron. 1976; 16:31-41.

11. Dindo D, Demartines N, Clavien PA. Classification of surgical complications. A new proposal with evaluation in a cohort of 6336 patients and results of a survey. Ann Surg. 2004; 240:205-13.

12. Citamak B, Altan M, Bozaci AC, et al. Percutaneous nephrolithotomy in children: 17 years of experience. J Urol. 2016; 195:1082-7.

13. Yadav P, Madhavan K, Syal S, et al. Technique, complications, and outcomes of pediatric urolithiasis management at a tertiary care hospital: evolving paradigms over the last 15 years. J Pediatr Urol. 2019; 15:665.e1-665.e7

14. Goyal NK, Goel A, Sankhwar SN, et al. A critical appraisal of complications of percutaneous nephrolithotomy in paediatric patients using adult instruments. BJU Int. 2014; 113:801-10.
15. Smaldone MC, Docimo SG, Ost MC. Contemporary surgical management of paediatric urolithiasis. Urol Clin North Am. 2010; 37:253-67.

16. Bilen CY, Kocak G, Kitirci O, et al. Percutaneous nephrolithotomy in children: lessons learned in 5 years at a single institution. $J$ Urol. 2007; 177:1867-71.

17. Jones P, Bennett G, Aboumarzouk OM, et al. Role of minimally invasive percutaneous nephrolithotomy techniques-micro and ultramini PCNL $(<15 F)$ in the pediatric population: a systematic review. J Endourol. 2017; 31:816-24.

18. Ozden E, Mercimek MN, Yakupoglu YK, et al. Modified Clavien classification in percutaneous nephrolithotomy: assessment of complications in children. J Urol. 2011; 185:264-8.

19. Mishra S, Sharma R, Garg C, et al. Prospective comparative study of miniperc and standard PNL for treatment of 1 to $2 \mathrm{~cm}$ size renal stone. BJU Int. 2011; 108:896-9.

20. Karatag T, Tepeler A, Silay MS, et al. Comparison of 2 percutaneous nephrolitotomy technique for the treatment of pediatric kidney stones of sized 10-20 mm: Microperc vs Miniperc. Urology. 2015; 85:015-8.

21. Onal B, Dogan HS, Satar N, et al. Factors affecting complication rates of percutaneous nephrolithotomy in children: results of a multi-institutional retrospective analysis by the Turkish pediatric urology society. J Urol. 2014; 191:777-82.

22. Altintas R, Oguz F, Tasdemir C, et al. The importance of instrument type in paediatric percutaneous nephrolithotomy. Urolithiasis. 2014; 42:149-53.

23. Traxer O, Smith $3^{\text {rd }} T G$, Pearle MS, et al. Renal parenchymal injury after standard and mini percutaneous nephrostolithotomy. J Urol. 2001; 165:1693-5.

24. Bayrak O, Erturhan S, Seckiner I, et al. Reliability of percutaneous nephrolithotomy in pediatric patients: comparison of complications with those in adults. Korean J Urol. 2013; 54:383-7.

25. Celik H, Camtosun A, Dede O, et al. Comparison of the results of pediatric percutaneous nephrolithotomy with different sized instruments. Urolithiasis. 2017; 45:203-8.

\section{Correspondence}

Volkan Izol, MD

Nihat Satar, MD

Yildirim Bayazit, MD

Nebil Akdogan, MD

Ibrahim Atilla Aridogan, MD

Department of Urology, Faculty of Medicine, University of Çukurova, Adana (Turkey)

Fatih Gokalp, MD, FEBU (Corresponding Author)

fatihgokalp85@gmail.com

Osmaniye Government Hospital, Urology Clinic, 80020,

Osmaniye (Turkey) 